\title{
Treatment of Platelet Deficiency in a Cohort of Patients by a Combination of Melatonin and 5-Methoxytryptamine
}

\section{Carlo Pastore*}

Clinica Villa Salaria, Rome

*Corresponding Author: Carlo Pastore, Clinica Villa Salaria, Rome.
Received: April 28, 2021

Published: June 23, 2021

(C) All rights are reserved by Carlo Pastore.

\begin{abstract}
This paper seeks to demonstrate how melatonin and 5-methoxytryptamine can affect the blood value of platelets in patients with low platelets count. Five Patients with ndd platelet loss were examined. After 6 months in all patients given a combination of melatonin and 5-methoxytryptamine, a significant increase in the value of platelets in hemochrome was observed
\end{abstract}

Keywords: Platelet Count; Melatonin; 5-methoxytryptamine

\section{Introduction}

Low platelet count is a clinical phenomenon that occurs in different pathological conditions and in particular during treatment in chemotherapy, advanced cancer pathology, myelofibrosis, septicaemia, autoimmune diseases [1]. In this paper we analyse a small cohort of patients diagnosed for essential low platelet count for whom the autoimmune hypothesis has been discarded. The aim of the work is to hypothesize a good activity of the combination of melatonin pineal hormones and 5-methoxytrriptamine in supporting medullar platelet production [2].

\section{Materials and Methods}

Five patients with essential platelet low count who had been treated in an ordinary way without results were enrolled and treated with the combination of melatonin at $20 \mathrm{mg}$ dosage in masterful preparation administered before going to sleep and 5-methoxytryptamine in masterfully prepared capsules administered in full daytime at $1 \mathrm{pm}$. The observation time was 6 months with complete hemochrome on a monthly basis after the first basal determination in order to follow the pattern of platelet counters.

\section{Results}

In all patients, a progressive increase in the platelet value with subjective oscillations has been observed. The best result was achieved in a 63 year old woman with an increase compared to the baseline value after six months of therapy of 138000 units (Figure 1). The therapy did not show any significant side effects.

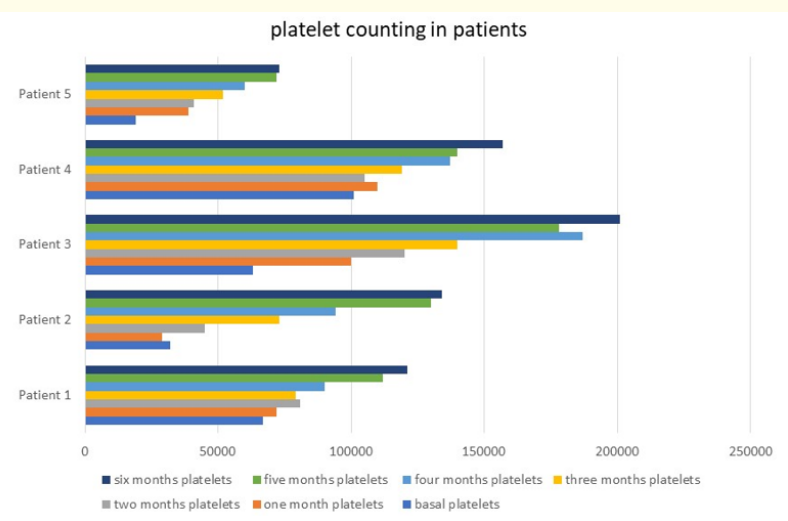

Figure 1: Development of platelet value in six months by administering a combination of melatonin and 5-methocytryptamine.

\section{Discussion}

In the international medical literature, an activity of modulation of the composition of the corpuscular elements of blood by 
epiphysial products is known [3]. In particular, the action of the gland on the concentration of platelets in the blood was studied. Often in daily clinical practice, particularly in medical oncology, we are faced with a platelet deficiency. In the specific condition often iatrogenic because antiblastic preparations have a particular haematological toxicity. Nevertheless, in other internal disciplines it is possible to deal with a decrease in the number of platelets [4-7]. The current pharmacological approach is mostly empirical, using corticosteroids derivatives and sometimes when platelet low count is also associated with anemia, erythropoietins, hoping for a stimulus activity between the red and platelet series [8]. A further possible treatment option without significant side-effects and low economic impact should be welcomed.

\section{Conclusion}

In this small cohort of patients in this pilot study, the use of two pituitary hormones has led to an increase in the number of circulating platelets. Numerical case studies should be increased and the pattern of platelet counting should be observed for longer periods of time.

\section{Bibliography}

1. Majerus P. Platelets, in The molecular basis of blood diseases, G Stamatoyanopoulis et al. Philadelphia, Saunders (1987): 689-722.

2. Lissoni P., et al. "Growth Factors: Thrombopoietic Property of the Pineal Hormone Melatonin". Hematology 4.4 (1999): 335343.

3. Di Bella L and Gualano L. "Key aspects of melatonin physiology: thirty years of research". Neuroendocrinology Letters 27.4 (2006): 425-432.

4. Buckley MF., et al. "A novel approach to the assessment of variations in the human platelet count". Thrombosis and Haemostasis 83.3 (2000): 480-484.

5. McMahon $\mathrm{CM}$ and Cuker A. "Hospital-acquired thrombocytopenia". Hospital Practice 42.4 (2014): 142-152.

6. Akca S., et al. "Time course of platelet counts in critically ill patients". Critical Care Medicine 30.4 (2002): 753-756.
7. Crowther MA., et al. "Thrombocytopenia in medical-surgical critically ill patients: prevalence, incidence, and risk factors". Journal of Critical Care 20.4 (2005): 348-353.

8. Kaushansky K. "Thrombopoiesis". Seminar on Hematology 52.1 (2015): 4-11.

Volume 5 Issue 7 July 2021

(C) All rights are reserved by Carlo Pastore. 